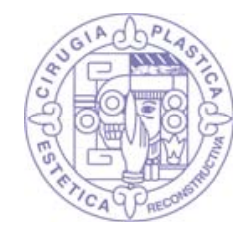

\title{
Neoformación del pezón con dos colgajos dermograsos en «L» opuestos
}

\author{
Neoformation of the nipple with two opposite «L» dermal fat flaps
}

Dr. Misael Contreras Marín,* Dr. Marco Antonio Barreda Gaxiola**

Palabras clave: Areola, pezón, reconstrucción.

Key words: Areola, nipple, reconstruction.

* Mayor Médico Cirujano, Especialista en Cirugía Plástica y Reconstructiva, adscrito al Servicio de Cirugía Plástica.

** Teniente Coronel Médico Cirujano,

Especialista en Cirugía Plástica y Reconstructiva Jefe del Servicio de Cirugía Plástica y

Reconstructiva.

Hospital Central Militar.

Los autores de este artículo no tienen conflicto de intereses qué declarar.

Recibido:

17 octubre 2017

Aceptado para

publicación:

7 mayo 2018
RESUMEN

El complejo pezón-areola se elimina junto con la piel que se reseca en una mastectomía y el objetivo de su reconstrucción es lograr una posición, simetría, color, tamaño, proyección y sensibilidad similar al de la mama sana. Ninguna técnica ha mostrado superioridad sobre otra de manera significativa, dos sobresalen por mantener una mejor proyección del neopezón, así como por lo simple y seguro de su realización (técnica en «patín de hielo» y el colgajo en $C-V)$. Sugerimos una nueva técnica en la neoformación del pezón con dos colgajos dermograsos en «L» opuestos. El objetivo es comparar en un modelo experimental una nueva técnica para formar el pezón con dos técnicas consideradas estándar de oro y medir la influencia de la neoformación de la areola, el tiempo, peso y estrés cutáneo en la conservación del nuevo pezón. Para ello se utilizaron seis cerdos de raza vietnamita, en cada uno se formaron de cada técnica dos nuevos pezones (con y sin areola). El seguimiento fue a ocho meses y los resultados se compararon con la prueba estadística ANOVA. No se encontraron diferencias estadísticamente significativas entre la técnica propuesta (dos colgajos en «L») y las técnicas conocidas (colgajo $\mathrm{C}-\mathrm{V}$ y en "patín de hielo») para la conservación del nuevo pezón $(p>0.05)$ y la técnica en «patín de hielo» fue superior en la proyección el pezón ( $\mathrm{p}$ $<0.05$ ). La posición del nuevo pezón y reconstrucción de la areola influyeron sobre la técnica en «patín de hielo» y colgajo $\mathrm{C}-\mathrm{V}$, respectivamente.

\section{ABSTRACT}

Nipple-areola complex is removed as part of the resected skin for a mastectomy, and the objective of its reconstruction is to achieve a position, symmetry, color, size, projection, and sensitivity similar to that of the healthy breast. No technique has been shown to be superior to another in a significant way, two stands out for maintaining a better projection of the neo-nipple, for simplicity and safety of their execution («ice skate» technique and flap in $C-V$ ). We suggest a new technique in the neoformation of the nipple with opposite double «L» dermal flaps. The objective is to compare a new technique of neoformation of the nipple with two techniques considered the gold standard in an experimental model. Six pigs of Vietnamese breed were used, in each one 2 neo-nipples of each technique were created (with areola and without areola). The followup was at 8 months and the results were compared with the ANOVA test. There were no statistically significant differences between the experimental technique (doublet «L» flaps) and the known techniques (C-V flap and «ice skate») for the preservation of the neo-nipple ( $p>0.05)$. The «ice skating» technique was superior in the nipple projection $(p<0.05)$ and the position of the neo-nipple and areola reconstruction influenced the «ice skate» and C-V flap techniques.

\section{INTRODUCCIÓN}

$\mathrm{D}$ e acuerdo con estimaciones de la Organización Mundial de la Salud, los casos de cáncer de mama aumentan en todo el mundo, con diferencias relevantes en la tasa de incidencia y el riesgo de morir, según sea la región geográfica. En Europa Occidental y Norteamérica, la mortalidad por cáncer de mama muestra una tendencia a la baja; en los países en desarrollo, sobre todo en América Latina, la incidencia y mortalidad se han incrementado de manera notoria. En México, desde el 2006 ocupa el primer lugar como causa de muerte por cáncer, siendo más importante en el norte y el centro del país, con tasas menores en los estados del sur. ${ }^{1}$ Es la segunda causa de muerte en mujeres de 30 a 54 años de edad y amenaza a todos los grupos socioeconómicos. Los datos sobre detección mostraron 6,000 casos nuevos en 1990 y se estima un incremento cercano a 16,500 casos nuevos anuales para $2020 .^{2}$ De acuerdo con los datos proporcionados por el Departamento de Estadística del 
Hospital Central Militar, se atendieron en esta institución 196 paciente con el diagnóstico de cáncer de mama en el periodo comprendido de noviembre de 2010 a diciembre de 2012, de los cuales 46 requirieron tratamiento quirúrgico y de éstos se les realizó un procedimiento reconstructivo a 13.

El ápex de la mama está formado por una zona redondeada y pigmentada: la areola y en su centro se encuentra el pezón. La areola es una zona regularmente circular de 4.2 a $4.5 \mathrm{~cm}$ de diámetro en promedio. Su color es variable: rosa en la mujer joven, que adquiere más tarde un color marrón, especialmente acentuado en el embarazo. El pezón se ubica en el centro de la areola: es cilíndrico o cónico, con una proyección promedio de 10 a $13 \mathrm{~mm}$ y diámetro aproximado de 4 a $9 \mathrm{~mm}$, pero en ocasiones está situado en el interior de una depresión excavada en la glándula subyacente (pezón umbilicado). Desde el punto de vista histológico, la piel de la areola está tapizada en profundidad por una capa de fibras musculares lisas, las que se insertan en la cara profunda de la dermis, constituida por una parte por fibras circulares dispuestas en anillos concéntricos y por otra por fibras radiadas. Por la constricción de estas fibras musculares debido al efecto de excitaciones diversas (toques, frío, emociones), la piel de la areola se contrae y proyecta el pezón hacia adelante (telotismo o erección del

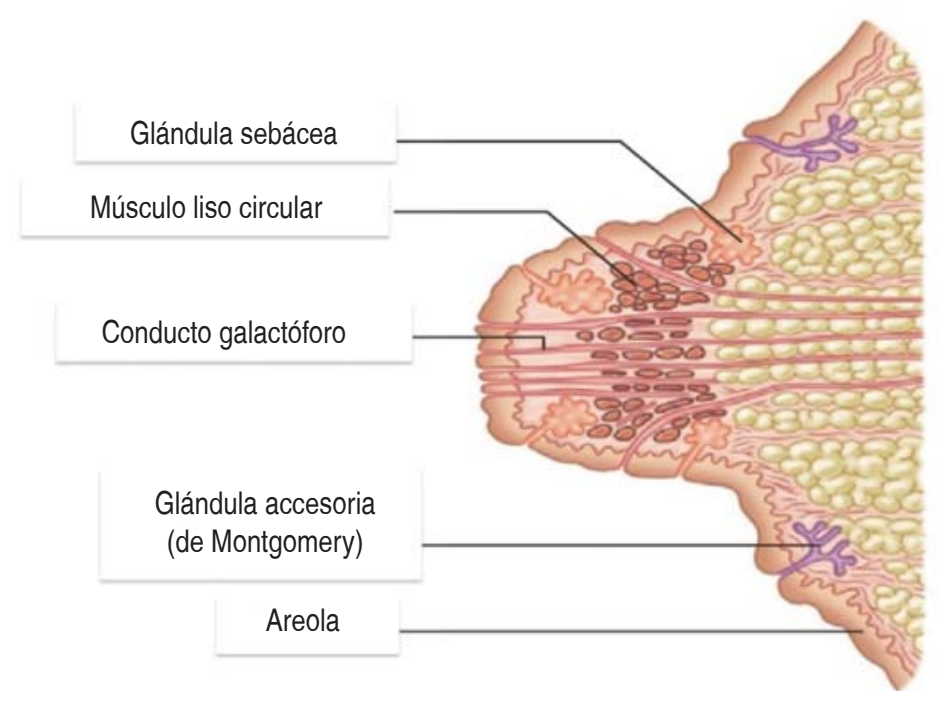

Figura 1. Anatomía del pezón. pezón); estas fibras musculares se continúan con el pezón y su contracción lo vuelve más pequeño y más duro en el curso del telotismo. Los conductos galactóforos atraviesan las fibras musculares del pezón y se abren en su ápex (Figura 1). ${ }^{3,4}$

El complejo areola-pezón (CAP) se extirpa rutinariamente junto con la piel que se reseca durante una mastectomía, sobre todo si el tumor se encuentra localizado en él y el objetivo de su reconstrucción es lograr una posición, simetría, color, tamaño, proyección y sensibilidad similar al de la mama sana. En lo que se refiere a la reconstrucción del pezón, se han ideado múltiples técnicas quirúrgicas. En 1944, Adams describió el trasplante de areola y pezón, mostrando preocupación por la incapacidad para mantener la proyección o de adquirir un color similar; esto motivó a otros a desarrollar opciones para la reconstrucción del complejo areola-pezón. ${ }^{5}$ Aunque la proyección es excelente en un inicio, todos sufren algún grado de aplanamiento con el paso del tiempo, reportándose en algunos estudios del 30 al $75 \%$ de pérdida de la proyección en un año; los colgajos en «patín de hielo» y en estrella son los que conservan más la proyección. ${ }^{6}$ Albert Losken y cols. ${ }^{7}$ reportaron en 2001 que el colgajo C-V (una modificación del colgajo en estrella descrita por Bostwick en 1994), conserva una proyección adecuada sin sufrir cambios estadísticamente significativos, en comparación con el pezón contralateral, con una satisfacción total del $81 \%$. En un estudio sobre la satisfacción de las pacientes después de la reconstrucción del CAP, Jarbor y cols. ${ }^{8}$ reportaron que el aplanamiento del pezón es la causa de insatisfacción hasta en más del 50\% de las mujeres encuestadas. Para superar la tendencia al aplanamiento del pezón reconstruido, se ha utilizado una variedad de materiales para aumentar la proyección del pezón, que incluye injertos de tejido graso subcutáneo, ${ }^{9}$ injerto de cartílago auricular, cartílago costal autólogo y alogénico liofilizado, ${ }^{10,11}$ infiltración de hidroxiapatita de calcio ${ }^{12}$ y AlloDerm para la reconstrucción primaria y secundaria, reportando una pérdida de la proyección del 44 al $53 \%$ en 12 meses. ${ }^{13,14}$ A pesar de los buenos resultados que tienen los colgajos locales, durante el tiempo hay una pérdida de la 
proyección y una contracción del tejido en un rango del 50 al 70\%, por lo que Di Bendetto y cols. proponen realizar una sobreproyección del neopezón en un rango del 50 al 60\% para lograr una simetría con el pezón contralateral. ${ }^{15}$ Una de las causas a las que se atribuye la pérdida de la proyección es la isquemia de los colgajos, siendo más susceptible a esto las mamas reconstruidas con técnicas que dejan una piel gruesa y tejido celular subcutáneo delgado, por lo que se recomienda efectuar una sobrecorrección del pezón en dichas mamas. ${ }^{16}$ Con base en lo anterior, se han introducido técnicas para reconstruir el CAP con doble pedículo vascular del colgajo que formará el pezón; el primero fue descrito por Cronin y cols. en 1988, denominándolo colgajo en «S»». Posteriormente, Kroll y Hamilton describieron en 1989 el colgajo «tap» en doble oposición y Ramakrishnan y cols. en 1997, describieron el colgajo gemelo. ${ }^{4,17}$ En 2008, Tatlidede y cols. ${ }^{18}$ describieron la técnica en domo con doble pedículo, manteniendo la proyección sin pérdida en un periodo de seguimiento de ocho a 12 meses con el uso de protectores durante los primeras tres semanas del postoperatorio. Otras técnicas prefieren colocar sutura no absorbible en forma de bolsa de tabaco alrededor de la areola para proyectar ligeramente la misma y ésta a su vez mejore la proyección del pezón, obteniendo pezones dentro del rango considerado como normal. ${ }^{19}$

De las técnicas existentes para neoformar el pezón, ninguna ha mostrado alguna superioridad sobre la otra en forma significativa; sin embargo, hay dos técnicas que sobresalen dentro de los colgajos locales por mantener una mejor proyección del neopezón y por la simpleza y seguridad de su realización; éstas son la técnica en «patín de hielo» y el colgajo en C-V. La pérdida de proyección es multifactorial y la isquemia potencial de los colgajos con la pérdida consecutiva de tejido es el principal factor, así como el proceso de cicatrización, la ausencia de tejido muscular subcutáneo, la localización de las incisiones en sentido diferente a las líneas de mínima tensión, el grosor de la piel, la tensión de los tejidos y el tipo de prendas de vestir que se utilizan después de la reconstrucción. Se sugiere una nueva técnica en la neoformación del pezón utilizando dos colgajos dermograsos en «L» opuestos, diseño del doctor Marco Antonio Barreda Gaxiola. Esta técnica provee dos colgajos con su respectivo aporte sanguíneo para mantener la proyección, forma y diámetro del neopezón; además, el pedículo tiene una relación 2:1 con la base del colgajo, en comparación con el colgajo en «patín de hielo» que es 5:1 y la base se somete a menos tensión durante el cierre, ya que se hace con una relación 1:1 con dicha base, al igual que con el colgajo en $\mathrm{C}-\mathrm{V}$; el colgajo en patín de hielo se cierra con una relación 2:1.

\section{MATERIAL Y MÉTODO}

Se utilizaron seis cerdos hembra de cinco meses de edad, no gestantes, no lactantes, sin enfermedades, con peso aproximado de $35 \mathrm{~kg}$. En cada cerdo se hicieron seis neopezones: dos con la técnica propuesta y dos de cada técnica a comparar. A un pezón de cada técnica se le colocó un injerto cutáneo para formar la areola, con la finalidad de observar la influencia del proceso de cicatrización en la base del neopezón sobre la conservación de éste. La distribución de cada neopezón y complejo areola-pezón formado en el dorso del cerdo fue variando entre cada uno de ellos, de manera que los seis sitios elegidos para cada neopezón fueron ocupados por las tres técnicas en sus dos formas: con areola y sin ella (Figura 2). Todos los pezones se midieron en el día 0 y en los meses 1, 2, 4, 6 y 8, para comparar los resultados.

La neoformación del pezón con dos colgajos dermograsos en «L» opuestos se realiza trazando una línea horizontal de $4 \mathrm{~cm}$, que se divide en cinco segmentos de $8 \mathrm{~mm}$ cada uno; en el tercer segmento se traza una línea perpendicular hacia arriba, de $8 \mathrm{~mm}$ en su borde izquierdo y una línea similar en dirección opuesta en el lado derecho. A continuación se traza una línea de $8 \mathrm{~mm}$, perpendicular a la línea principal dirigida hacia abajo, a nivel de la unión del primero y segundo segmento, que a su vez se proyecta hacia el punto inicial de la línea principal para formar un triángulo equilátero hacia el nivel de unión del segundo con el tercer segmento en dirección paralela a la línea principal, quedando una distancia de $8 \mathrm{~mm}$ libre entre esta última línea y la línea 
Figura 2.

Distribución de los pezones en el dorso de cada cerdo.
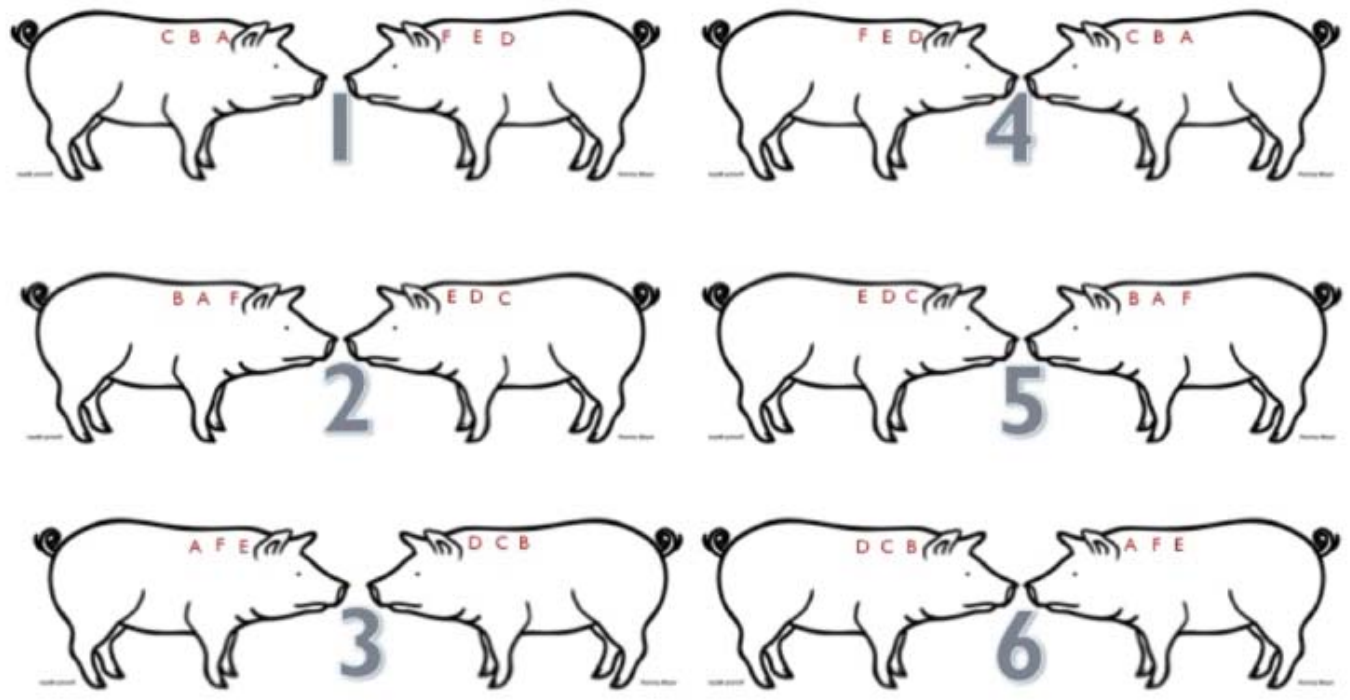

perpendicular a la principal, formando así la base del primer colgajo en «L». Se procede de la misma forma pero en dirección opuesta y hacia arriba para crear el segundo colgajo en «L» (Figura 3A). A continuación, se incide sobre las líneas marcadas incluyendo piel y tejido celular subcutáneo, desepitelizando el área triangular de los extremos para obtener un injerto de piel de espesor total, levantando así los dos colgajos en «L» (Figura 3B). Ambos colgajos se afrontan en doble oposición con puntos simples de nylon 3-0 para formar el neopezón (Figura 3C). Una vez formado el pezón se coloca sobre su domo el injerto de piel de espesor total previamente obtenido, fijándolo con puntos simples de nylon 3-0 en borde superior de los colgajos (Figura 3D).

\section{RESULTADOS}

En la proyección del pezón reconstruido con la técnica experimental (dos colgajos en «L») comparada con las técnicas conocidas (colgajo $\mathrm{C}-\mathrm{V}$ y en «patín de hielo»), encontramos que donde se reconstruyó la areola no hubo diferencias estadísticamente significativas ( $p>$ 0.05) en los diferentes tiempos de medición, sólo en el tiempo 0 se encontró diferencia entre la técnica de dos colgajos en «L» y la técnica en «patín de hielo» ( $p<0.05)$. De igual manera, pero sin reconstrucción de la areola, hubo diferencias entre la técnica de dos colgajos en
«L» con la técnica en «patín de hielo» en todos los tiempos de medición, con excepción del tiempo 1 (Cuadro I).

En el diámetro del pezón reconstruido con la técnica experimental y las técnicas conocidas encontramos que donde se reconstruyó la areola no hubo diferencias estadísticamente significativas $(p>0.05)$ en los diferentes tiempos de medición y donde no se reconstruyó la areola no hubo diferencias, con excepción del tiempo de medición 1 con el colgajo C-V ( $\mathrm{p}=$ 0.06) (Cuadro I).

En el volumen del pezón reconstruido encontramos que donde se reconstruyó la areola no hubo diferencias significativas ( $p>0.05$ ) en los diferentes tiempos de medición, sólo en el tiempo 0, en el que hubo diferencia entre la técnica de dos colgajos en «L» y la técnica en «patín de hielo» $(\mathrm{p}<0.05)$ y donde no se reconstruyó la areola no hubo diferencias significativas ( $p>0.05)$ en los diferentes tiempos de medición, sólo en los tiempos 0 y 2, en los que se observó diferencia entre la técnica de dos colgajos en «L» y la técnica en «patín de hielo» $(\mathrm{p}<0.05)$ (Cuadro I).

\section{DISCUSIÓN}

El pezón es un punto de referencia anatómica bien definido que contribuye significativamente al resultado estético final en el proceso reconstructivo de la mama. Se han realizado muchos 

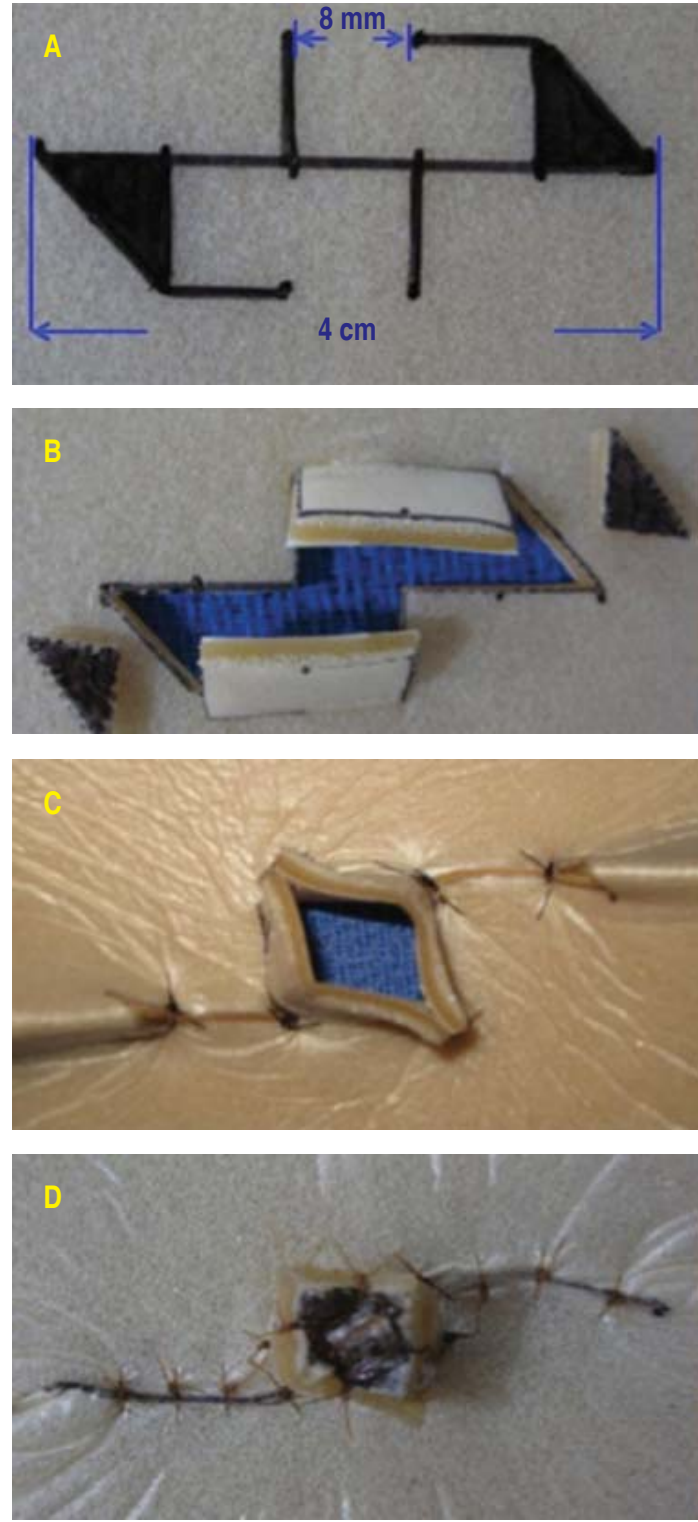
en «L» en oposición.

Técnica de dos colgajos dermograsos

estudios con las diferentes técnicas que existen hasta este momento para reconstruir el complejo areola-pezón con resultados variables. Todos coinciden en que el principal desafío hasta el momento sigue siendo la pérdida de la proyección del pezón con el paso del tiempo, que dependiendo del autor va del $30 \%$ hasta el $75 \%, 6,15$ sin encontrar una mejoría significativa al combinar esas técnicas con el uso de injertos de grasa, injertos de cartílago, aplicación de hidroxiapatita de calcio o AlloDerm, reportando con dicho uso una pérdida de la proyección del neopezón del 44 al 53\%. ${ }^{10-14}$
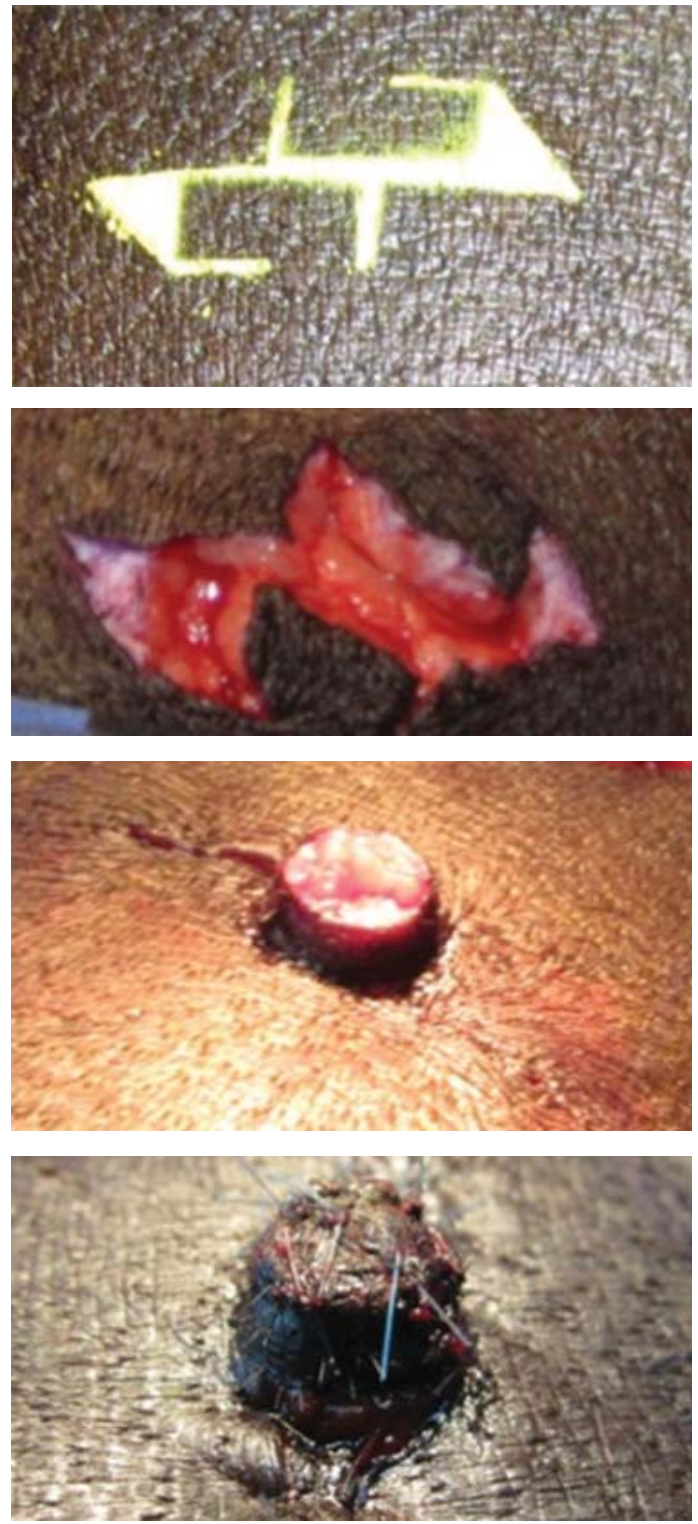

Sin embargo, estos estudios se han realizado en pacientes de diferentes edades (rango de 25 a 77 años) que se han sometido a diferentes tipos de reconstrucción del montículo mamario (colgajo TRAM, colgajo de músculo dorsal ancho, o expansión tisular con colocación de implantes), así como a diferentes tratamientos adyuvantes para el cáncer de mama (quimioterapia y/o radioterapia). Estas variables alteran el resultado que se obtiene de cada técnica estudiada, además de que algunos de esos estudios pierden valor al reportar pocos casos y tener un pobre cumplimiento de las encuestas. 
Hasta el momento no existe en la literatura un estudio experimental donde todas las técnicas estudiadas se realicen en el mismo grupo de pacientes y bajo las mismas circunstancias. Por esta razón, la técnica que proponemos la comparamos en un modelo experimental (cerdos hembra de raza vietnamita productos de la misma camada) con las dos técnicas que al momento se pueden considerar como estándar de oro en la reconstrucción de CAP por sus

\begin{tabular}{|c|c|c|c|c|c|c|c|}
\hline \multirow{2}{*}{$\begin{array}{l}\text { Parámetro } \\
\text { medido }\end{array}$} & \multirow{2}{*}{$\begin{array}{l}\text { Tipo de } \\
\text { reconstrucción }\end{array}$} & \multirow{2}{*}{$\begin{array}{c}\text { Tiempo de } \\
\text { medición (mes) }\end{array}$} & \multirow{2}{*}{$\begin{array}{c}\text { Técnica } \\
\text { experimental }\end{array}$} & \multicolumn{4}{|c|}{ Técnicas conocidas } \\
\hline & & & & Colgajo C-V & Valor $\mathrm{p}$ & Patín de hielo & Valor $\mathrm{p}$ \\
\hline \multirow[t]{12}{*}{ Proyección } & \multirow{6}{*}{$\begin{array}{l}\text { Con reconstrucción } \\
\text { de areola }\end{array}$} & 0 & $9 \pm 2.53$ & $8.33 \pm 0.51$ & NS & $12.83 \pm 2.78$ & 0.005 \\
\hline & & 1 & $3.67 \pm 1.21$ & $4.5 \pm 2.42$ & NS & $6.17 \pm 3.37$ & NS \\
\hline & & 2 & $3.67 \pm 1.21$ & $3.83 \pm 1.60$ & NS & $5.17 \pm 1.60$ & NS \\
\hline & & 4 & $3.17 \pm 1.72$ & $3.17 \pm 1.94$ & NS & $4.33 \pm 1.21$ & NS \\
\hline & & 6 & $2.50 \pm 1.76$ & $2.67 \pm 1.75$ & NS & $3.17 \pm 0.75$ & NS \\
\hline & & 8 & $2.33 \pm 1.96$ & $2.67 \pm 1.75$ & NS & $3.17 \pm 0.75$ & NS \\
\hline & \multirow{6}{*}{$\begin{array}{l}\text { Sin reconstrucción } \\
\text { de areola }\end{array}$} & 0 & $7.76 \pm 1.21$ & $8.33 \pm 1.36$ & NS & $13.11 \pm 1.96$ & 0.0001 \\
\hline & & 1 & $4.33 \pm 1.63$ & $6.0 \pm 1.78$ & NS & $7.67 \pm 2.94$ & 0.06 \\
\hline & & 2 & $3.83 \pm 1.16$ & $4.33 \pm 0.81$ & NS & $6.83 \pm 2.7$ & 0.01 \\
\hline & & 4 & $3.17 \pm 1.6$ & $3.33 \pm 1.36$ & NS & $6.17 \pm 3.12$ & 0.03 \\
\hline & & 6 & $2.83 \pm 1.32$ & $2.17 \pm 0.75$ & NS & $5.83 \pm 3.06$ & 0.01 \\
\hline & & 8 & $2.83 \pm 1.32$ & $1.83 \pm 0.98$ & NS & $5.67 \pm 3.26$ & 0.04 \\
\hline \multirow{12}{*}{ Diámetro } & \multirow{6}{*}{$\begin{array}{l}\text { Con reconstrucción } \\
\text { de areola }\end{array}$} & 0 & $12 \pm 2.19$ & $11 \pm 1.54$ & NS & $12.5 \pm 1.37$ & NS \\
\hline & & 1 & $12.83 \pm 0.98$ & $11.33 \pm 1.03$ & NS & $12.17 \pm 1.16$ & NS \\
\hline & & 2 & $12.83 \pm 0.98$ & $11.5 \pm 1.04$ & NS & $11.33 \pm 1.50$ & NS \\
\hline & & 4 & $13 \pm 2.09$ & $11.67 \pm 1.36$ & NS & $11.33 \pm 1.36$ & NS \\
\hline & & 6 & $13 \pm 1.09$ & $12 \pm 1.67$ & NS & $11 \pm 1.67$ & NS \\
\hline & & 8 & $13 \pm 1.09$ & $12 \pm 1.67$ & NS & $11 \pm 1.67$ & NS \\
\hline & \multirow{6}{*}{$\begin{array}{l}\text { Sin reconstrucción } \\
\text { de areola }\end{array}$} & 0 & $13 \pm 0.63$ & $11.17 \pm 1.16$ & NS & $12.17 \pm 1.94$ & NS \\
\hline & & 1 & $12.67 \pm 1.03$ & $10.83 \pm 0.98$ & 0.06 & $12 \pm 2.09$ & NS \\
\hline & & 2 & $11.67 \pm 1.86$ & $10.83 \pm 1.03$ & NS & $11.67 \pm 1.96$ & NS \\
\hline & & 4 & $11.67 \pm 2.25$ & $10.83 \pm 1$ & NS & $11.83 \pm 2.22$ & NS \\
\hline & & 6 & $11.5 \pm 2.42$ & $10 \pm 1.41$ & NS & $12 \pm 2.09$ & NS \\
\hline & & 8 & $11.5 \pm 2.42$ & $10 \pm 1.41$ & NS & $12.33 \pm 2.33$ & NS \\
\hline \multirow[t]{12}{*}{ Volumen } & \multirow{6}{*}{$\begin{array}{l}\text { Con reconstrucción } \\
\text { de areola }\end{array}$} & 0 & $0.97 \pm 0.22$ & $0.8 \pm 0.21$ & NS & $1.57 \pm 0.42$ & 0.009 \\
\hline & & 1 & $0.48 \pm 0.20$ & $0.47 \pm 0.28$ & NS & $0.73 \pm 0.49$ & NS \\
\hline & & 2 & $0.48 \pm 0.20$ & $0.41 \pm 0.19$ & NS & $0.50 \pm 0.15$ & NS \\
\hline & & 4 & $0.35 \pm 0.24$ & $0.35 \pm 0.21$ & NS & $0.42 \pm 0.14$ & NS \\
\hline & & 6 & $0.20 \pm 0.13$ & $0.32 \pm 0.22$ & NS & $0.30 \pm 0.12$ & NS \\
\hline & & 8 & $0.17 \pm 0.15$ & $0.26 \pm 0.20$ & NS & $0.30 \pm 0.12$ & NS \\
\hline & \multirow{6}{*}{$\begin{array}{l}\text { Sin reconstrucción } \\
\text { de areola }\end{array}$} & 0 & $1 \pm 0.1$ & $0.79 \pm 0.11$ & NS & $1.59 \pm 0.55$ & 0.01 \\
\hline & & 1 & $0.55 \pm 0.23$ & $0.56 \pm 0.28$ & NS & $0.9 \pm 0.46$ & NS \\
\hline & & 2 & $0.41 \pm 0.19$ & $0.36 \pm 0.09$ & NS & $0.76 \pm 0.41$ & 0.04 \\
\hline & & 4 & $0.35 \pm 0.25$ & $0.26 \pm 0.09$ & NS & $0.54 \pm 0.28$ & NS \\
\hline & & 6 & $0.32 \pm 0.26$ & $0.17 \pm 0.1$ & NS & $0.52 \pm 0.25$ & NS \\
\hline & & 8 & $0.32 \pm 0.26$ & $0.14 \pm 0.09$ & NS & $0.52 \pm 0.27$ & NS \\
\hline
\end{tabular}


resultados obtenidos y facilidad de ejecución (colgajo en «patín de hielo» y colgajo en C-V).

En nuestros resultados pudimos observar que al someter las tres técnicas a las mismas condiciones, el porcentaje de pérdida en la conservación del pezón fue mayor que lo reportado por los diferentes autores, ya que el colgajo en C-V tuvo una pérdida de la proyección que varió del 37.5 al $100 \%$ (dos casos), con una media de $70.19 \%$ y una pérdida de volumen del pezón del 28.2 al $100 \%$ (dos casos) con una media de $73.26 \%$. De igual forma, con la técnica en «patín de hielo» se observó una pérdida de proyección del pezón del 23 al $85 \%$, con una media de $61.5 \%$ y una pérdida del volumen del pezón del 40.3 al 90\%, con una media de $68 \%$.

La técnica con dos colgajos dermograsos en «L» tuvo una pérdida de la proyección del pezón del 28.5 al 100\% (un caso), con una media de $60.9 \%$ y una pérdida en el volumen del pezón del 17.3 al 100\% (un caso), con una media de $68.8 \%$, ubicándose a la par del colgajo en «patín de hielo» y por arriba del colgajo en C-V. Además, la técnica propuesta no presentó cambios significativos en la reconstrucción de la areola con injerto de piel de espesor total ni con el sitio de localización, a diferencia de las otras dos técnicas; la del colgajo en C-V tuvo diferencias significativas al agregarle la reconstrucción de la areola y el colgajo en «patín de hielo» al ubicarlo en sitios de tensión. No obstante, con el análisis estadístico utilizado (ANOVA), la diferencia entre la técnica propuesta y las otras dos no fue significativa para la conservación del volumen y diámetro del pezón, demostrando con esto que el resultado sigue siendo malo en la conservación del neopezón, independientemente de la técnica utilizada; sólo la técnica en «patín de hielo» fue superior al mantener una mayor proyección. Cabe mencionar que con esta técnica el neopezón se encuentra muy proyectado desde el inicio, incluso se puede considerar deforme para simular un pezón, no así con las otras dos técnicas.

Como bien lo comentan J. Farhadi y cols., ${ }^{17}$ todos los tejidos que se han utilizado para reconstruir el CAP carecen de los elementos estructurales de un pezón normal, es decir, músculo liso y conductos galactóforos, que son los principales responsables de la firmeza natural y proyección del pezón. Por esta razón las técnicas futuras deben buscar reconstruir esta anatomía que hasta el momento ninguna de las técnicas propuestas ha logrado y que consideramos imposible de realizar.

\section{CONCLUSIONES}

Con base en los resultados obtenidos en este estudio, concluimos que la neoformación del pezón con dos colgajos dermograsos en «L» en oposición es una nueva herramienta para la reconstrucción del CAP, fácil de realizar y diseñar y brinda resultados similares a los que se obtienen con las técnicas que se consideran prácticamente estándar de oro (colgajo en «patín de hielo» y colgajo en C-V).

La neoformación de la areola con injerto de piel de espesor parcial no influye en la conservación del pezón para las técnicas con dos colgajos en «L»y en «patín de hielo», condicionando un aumento en el diámetro del pezón y una menor pérdida de proyección a la técnica con colgajo en C-V.

La posición no modifica la conservación del pezón para las técnicas con dos colgajos en «L» y en C-V. La técnica en «patín de hielo» lleva a un aumento del diámetro del pezón en áreas de tensión.

El colgajo en «patín de hielo» sigue siendo la técnica para reconstruir el CAP que conserva una mayor proyección del pezón, no así la forma y el volumen.

El modelo experimental propuesto posee características similares a las de una mama reconstruida, así como las condiciones que afectan al pezón neoformado en el postoperatorio (tiempo, peso y estrés cutáneo).

Ninguna de las tres técnicas tiene resultados satisfactorios.

\section{REFERENCIAS}

1. Castillo-Gutiérrez M, Ávila-Esquivel JF. Cáncer de mama: etapa clínica en la que se realiza el diagnóstico y tipos histológicos más frecuentes en mujeres que acuden a un hospital de segundo nivel. Arch Inv Mat Inf 2010; 2 (2): 85-90.

2. Knaul FM, Nigenda G, Lozano R, Arreola-Ornelas H, Langer A, Frenk J. Cáncer de mama en México: una prioridad apremiante. Salud Pública Mex 2009; 51 Supl 2: S335-S344.

3. Latarjet M, Ruiz-Liard A. Anatomía humana. 3a ed. España: Panamericana; 1999. Vol. II, pp. 1779-1786. 
4. Neligan PC. Plastic surgery. 3rd ed. Elsevier; 2013. Vol. 5, pp. 1-12.

5. Stephen JM, Cristiane MU. Plastic surgery. 2nd ed. Elsevier; 2006. Vol. VI, pp. 791-818.

6. Shestak KC, Gabriel A, Landecker A, Peters S. Assessment of long-term nipple projection: a comparison of three techniques. Plast Reconstr Surg 2002; 110 (3): 780-786.

7. Losken A, Mackay GJ, Bostwick J. Nipple reconstruction using the $\mathrm{C}-\mathrm{V}$ flap technique: a long-term evaluation. Plast Reconstr Surg 2001; 108 (2): 361-369.

8. Jabor MA, Shayani P, Collins Jr. DR, Karas T, Cohen BE. Nipple-areola reconstruction: satisfaction and clinical determinants. Plast Reconstr Surg 2002; 110 (2): 457-463.

9. Bernard RW, Beran SJ. Autologous fat graft in nipple reconstruction. Plast Reconstr Surg 2003; 112 (4): 964-968.

10. Guerra AB, Khoobehi K, Metzinger SE, Allen RJ. New technique for nipple areola reconstruction: arrow flap and rib cartilage graft for long-lasting nipple projection. Ann Plast Surg 2003; 50 (1): 31-37.

11. Kim EK, Lee TJ. Use of lyophilized allogenic costal cartilage: is it effective to maintain the projection of the reconstructed nipple? Ann Plast Surg 2011; 66 (2): 128-130.

12. Evans KK, Rasko Y, Lenert J, Olding M. The use of calcium hydroxyapatite for nipple projection after failed nipple-areolar reconstruction. Ann Plast Surg 2005; 55 (1): 25-29.

13. Garramone CE, Lam B. Use of AlloDerm in primary nipple reconstruction to improve long-term nipple projection. Plast Reconstr Surg 2007; 119 (6): 1663-1667.
14. Nahabedian MY. Secondary nipple reconstruction using local flaps and AlloDerm. Plast Reconstr Surg 2005; 115 (7): 2056-2061.

15. Di Benedetto G, Sperti V, Pierangeli M, Bertani A. A simple and reliable method of nipple reconstruction using a spiral flap made of residual scar tissue. Plast Reconstr Surg 2004; 114 (1): 158-160.

16. Hammond DC, Khuthaila D, Kim J. The skate flap purse-string technique for nipple-areola complex reconstruction. Plast Reconstr Surg 2007; 120 (2): 399-406.

17. Farhadi J, Maksvytyte GK, Schaefer DJ, Pierer G, Scheufler O. Reconstruction of the nipple-areola complex: an update. J Plast Reconstr Aesth Surg 2006; 59: 40-53.

18. Tatlidede S, Yesilada AK, Egemen O, Bas L. A new technique in nipple reconstruction: dome technique with double pedicle. Ann Plast Surg 2008; 60 (2): 141-143.

19. Weinfeld AB, Somia N, Codner MA. Purse-string nipple areolar reconstruction. Ann Plast Surg 2008; 61 (4): 364-367.

\section{Correspondencia:}

\section{Dr. Misael Contreras Marín}

Hospital Central Militar

Av. Manuel Ávila Camacho S/N,

Col. Lomas de Sotelo, 11200,

Del. Miguel Hidalgo,

Ciudad de México, México

E-mail: contacto@drmisaelcm.com 